\title{
Localized modes of the Hirota equation: $N$-th order rogue wave and a separation of variable technique
}

\author{
Gui $\mathrm{Mu}^{1}$, Zhenyun Qin ${ }^{2}$, Kwok Wing Chow ${ }^{3}$ and Bernard K. Ee ${ }^{4}$ \\ ${ }^{1}$ College of Mathematics and Information Science, Qujing Normal University, Qujing \\ 655011, PR China \\ 2 School of Mathematics and LMNS, Fudan University, Shanghai 200433, PR China \\ ${ }^{3}$ Department of Mechanical Engineering, University of Hong Kong, Pokfulam, Hong \\ Kong \\ ${ }^{4}$ School of Mechanical Engineering, Tel Aviv University, Tel Aviv 69978, Israel
}

\begin{abstract}
The Hirota equation is a special extension of the intensively studied nonlinear Schrödinger equation, by incorporating third order dispersion and one form of the self-steepening effect. Higher order rogue waves of the Hirota equation can be calculated theoretically through a Darboux-dressing transformation by a separation of variable approach. A Taylor expansion is used and no derivative calculation is invoked. Furthermore, stability of these rogue waves is studied computationally. By tracing the evolution of an exact solution perturbed by random noise, it is found that second order rogue waves are generally less stable than first order ones.
\end{abstract}

Keywords: Rogue waves, Variable separation technique, Generalized Darboux transformation, Stability of rogue waves

\section{Introduction}

Solitons and rogue waves are widely studied nonlinear modes for evolution equations in the past few years. Solitons are permanent propagating modes and as such there will always be a finite displacement for all values in time. In contrast, rogue waves are localized in both space and time. In other words, a rogue wave can only be detected within a finite interval in time, a feature which highlights the difference between a rogue wave and a soliton. While such rogue waves have been known in the maritime community for over a century, intensive scientific interests only started about twenty years 
ago. In the deep ocean, such large amplitude displacements from an otherwise calm background state obviously pose threat to shipping and offshore platforms $[2,3]$. In other aspects of hydrodynamic surface waves, such nonlinear entities also arise in the regime of shallow water waves [4], where the theory of hyperbolic systems demonstrates the importance of wave-boundary interactions. Furthermore, the presence of a current will have a significant effect in the statistical description of rogue waves [5]. In many other fields of applied sciences, rogue waves occur too and are commanding increasing attention, e.g. space physics [6], optics [7], plasma physics [8], superfluids and Bose-Einstein condensates [9, 10].

The precise mechanisms for the generation of rogue waves are still not completely understood, but contributions from various complex processes, e.g. dispersion enhancement of transient wave groups, geometric focusing, wave current interactions as well as modulation (Benjamin-Feir) instability are believed to be relevant [11]. The most commonly used theoretical model is the nonlinear Schrödinger equation (NLSE)

$$
\mathrm{i} u_{t}+\frac{1}{2} u_{x x}+|u|^{2} u=0,
$$

which incorporates a second order dispersion and a cubic nonlinearity. The NLSE has been well established as the governing equation for slowly varying wave packets for deep water waves and optical waveguides $[12,13]$. The widely employed example of a rogue wave is the exact algebraic breather on a plane wave background, also frequently termed the Peregrine breather [14]. The maximum amplitude of this rogue wave is three times that of the background plane wave.

Higher order rogue waves of the NLSE were calculated analytically by the Darboux transformation [15]. These higher order units may have an amplitude higher than that of the fundamental rogue wave. The Hirota bilinear transformation, a firmly established method in soliton theory, can also be utilized to find rogue waves of the NLSE in terms of determinants [16]. The connections among eigenvalues, finite gap integration and rogue waves have been examined [17]. Higher order rogue waves can be calculated by progressive fusion and fission of degenerate breathers [18].

In the context of optical waveguides, third order dispersion and selfsteeping nonlinearity might need to be taken into account for sufficiently short pulses [13]. While many such equations do not possess analytical solutions, a few models permit theoretical advances and will provide valuable 
physical insight. We shall focus on the Hirota equation

$$
\mathrm{i} u_{t}+\frac{1}{2} u_{x x}+|u|^{2} u+\mathrm{i} \alpha\left(u_{x x x}+6|u|^{2} u_{x}\right)=0 .
$$

The physical interpretations of $u, x, t$, and $\alpha$ in an optical fiber context are complex valued slowly varying envelope, delayed time, space propagation variable and relative magnitude of third order dispersion respectively. The two lowest order rogue waves have been given explicitly by the Darboux transformation [19]. However, an improved version of the Darboux transformation is derived in Section 3. This new separation of variable technique will allow the rogue waves to be obtained without any calculations of the derivatives. Using a parameterized Darboux transformation, higher order, rational rogue waves can be obtained [18, 20].

The goal of the present study is to conduct further studies into the rogue wave modes of the Hirota equation. More precisely, a variable separation technique will be introduced to solve the Lax pair of the Hirota equation. Fully explicit representation of the rogue waves can be generated.

The outline of the present work can now be described. The variable separation technique to treat the Lax pair of the Hirota equation will be described in Section 2. Rogue waves of arbitrary order are established by a Taylor expansion mechanism in Section 3. The two lowest order rogue waves are computed as illustrative examples and illustrate a range of dynamic behaviours of our obtained third order rogue wave solutions is displayed in Section 4. Computational studies of stability are conducted in Section 5. Further extensions and challenges are highlighted in the final section.

\section{A variable separation technique}

Eq. (2) is integrable; and the associated Lax pair is

$$
\begin{aligned}
& \Psi_{x}=(\lambda \sigma+Q) \Psi, \\
& \Psi_{t}=\left[4 \alpha \lambda^{3} \sigma+\lambda^{2}(\sigma+4 \alpha Q)+\lambda\left(-2 \alpha|u|^{2} \sigma+Q+2 \alpha \mathrm{i} V_{1}\right)+V_{2}\right] \Psi,
\end{aligned}
$$

where $\Psi(x, t)$ is a $2 \times 2$ matrix solution, $\lambda$ is the complex spectral variable, $\sigma$ is a diagonal matrix with entries $-\mathrm{i}$ and $\mathrm{i}$, and $Q=Q(x, t)$ is the $2 \times 2$ 
matrix

$$
\begin{aligned}
Q & =\left(\begin{array}{cc}
0 & u \\
-u^{*} & 0
\end{array}\right), V_{1}=\left(\begin{array}{cc}
0 & u_{x} \\
-u_{x}^{*} & 0
\end{array}\right), \\
V_{2} & =-\frac{1}{2}|u|^{2} \sigma+\frac{1}{2} \mathrm{i} V_{1}+\alpha\left(\begin{array}{cc}
u u_{x}^{*}-u^{*} u_{x} & -u_{x x}-|u|^{2} u \\
-u_{x x}^{*}-|u|^{2} u^{*} & u u_{x}^{*}-u^{*} u_{x}
\end{array}\right) .
\end{aligned}
$$

By direct analysis, Eq. (2) admits the following seed solution

$$
u=\exp (\mathrm{i} t) \text {. }
$$

We seek a family of the solutions of the Lax system (3) corresponding to the spectral parameter $\lambda$ in the following form

$$
\begin{aligned}
& \Psi=\left(\begin{array}{l}
\psi(x, t) \\
\phi(x, t)
\end{array}\right)=A F G Z, \\
& F=\exp (\mathrm{i} \Lambda x), \quad G=\exp (\mathrm{i} \Omega t),
\end{aligned}
$$

where

$$
A=\left(\begin{array}{cc}
1 & 0 \\
0 & e^{ \pm \mathrm{i} \zeta}
\end{array}\right)
$$

with $\zeta=k x+c t$ and $Z$ is an arbitrary complex two dimensional vector. Here, it is assumed that

$$
[\Lambda, \Omega]=\Lambda \Omega-\Omega \Lambda=0 .
$$

Inserting (7) into (3) yields

$$
A_{x}+\mathrm{i} A \Lambda-U A=0, \quad A_{t}+\mathrm{i} A \Omega-V A=0 .
$$

Solving conditions (9) and (10), we have

$$
\begin{aligned}
& A=\left(\begin{array}{cc}
1 & 0 \\
0 & e^{-\mathrm{i} t}
\end{array}\right), \quad \Lambda=\left(\begin{array}{cc}
-\lambda & -\mathrm{i} \\
\mathrm{i} & \lambda
\end{array}\right), \\
& \Omega=\left(4 \alpha \lambda^{2}+\lambda-2 \alpha\right) \Lambda+\frac{1}{2} .
\end{aligned}
$$

Hence, $F$ can be rewritten as

$$
F=\frac{1}{2 \eta}\left(\begin{array}{cc}
-2 \mathrm{i} \lambda \sin (\eta x)+2 \eta \cos (\eta x) & 2 \sin (\eta x) \\
-2 \sin (\eta x) & 2 \mathrm{i} \lambda \sin (\eta x)+2 \eta \cos (\eta x)
\end{array}\right),
$$

where $\eta=\sqrt{1+\lambda^{2}}$. 


\section{Derivation of $N$ th order rogue waves}

In this section we concentrate on producing higher order rogue wave solutions of equation (2). Let $\lambda=\mathrm{i}(1+\delta)$ and use the following Taylor expansion formulas

$$
\sin (x)=\sum_{n=0}^{\infty}(-1)^{n} \frac{x^{2 n+1}}{(2 n+1) !}, \quad \cos (x)=\sum_{n=0}^{\infty}(-1)^{n} \frac{x^{2 n}}{(2 n) !},
$$

the matrix $F$ has the expansion at $\delta=0$ as

$$
\left.F\right|_{\lambda=\mathrm{i}(1+\delta)}=\sum_{n=1}^{\infty} F_{n} \delta^{n}
$$

where

$$
F_{n}=\left(\begin{array}{cc}
\gamma_{n}+\tau_{n}+\tau_{n-1} & \tau_{n} \\
-\tau_{n} & \gamma_{n}-\tau_{n}-\tau_{n-1}
\end{array}\right)
$$

with

$$
\gamma_{n}=\sum_{l=0}^{\lfloor n / 2\rfloor} C_{n-l}^{l} 2^{n-2 l} X_{2(n-l)}, \quad \tau_{n}=\sum_{l=0}^{\lfloor n / 2\rfloor} C_{n-l}^{l} 2^{n-2 l} X_{2(n-l)+1}, \quad X_{m}=\frac{x^{m}}{m !} .
$$

Hereafter, we use the notation $C_{n}^{m}=\frac{n !}{m !(n-m) !}$, where $n$ and $m$ are nonnegative integers and $n \geq m$.

Similarly, the matrix $G$ can be decomposed into a product of three matrices,

$$
\begin{aligned}
& G=\exp (\mathrm{i} t / 2) \Theta \Pi \Upsilon, \quad \Theta=\exp (-2 \mathrm{i} \alpha \Lambda t), \\
& \Pi=\exp (\mathrm{i} \lambda \Lambda t), \quad \Upsilon=\exp \left(4 \mathrm{i} \alpha \lambda^{2} \Lambda t\right)
\end{aligned}
$$

We postulate that $\Theta, \Pi, \Upsilon$ have series expansions at $\delta=0$ as

$$
\Theta=\sum_{n=1}^{\infty} \Theta_{n} \delta^{n}, \quad \Pi=\sum_{n=1}^{\infty} \Pi_{n} \delta^{n}, \quad \Upsilon=\sum_{n=1}^{\infty} \Upsilon_{n} \delta^{n}
$$

Thus, the matrix $G$ has the expansion at $\delta=0$ as

$$
\left.G\right|_{\lambda=\mathrm{i}(1+\delta)}=\sum_{n=1}^{\infty} G_{n} \delta^{n}
$$


and the matrix $G_{n}$ is obtained as follows

$$
G_{n}=\sum_{k=0}^{n} \sum_{j=0}^{n} \Theta_{k} \Pi_{j} \Upsilon_{n-k-j} .
$$

In fact, the matrix $\Theta_{n}$ is easily obtained after replacing $x$ by $-2 \alpha t$ in the matrix $F_{n}$ while the matrix $\Pi_{n}$ is given by

$$
\Pi_{n}=\left(\begin{array}{cc}
\rho_{n}+\mathrm{i} \chi_{n}+\mathrm{i} \chi_{n-1} & \mathrm{i} \chi_{n} \\
-\mathrm{i} \chi_{n} & \rho_{n}-\mathrm{i} \chi_{n}-\mathrm{i} \chi_{n-1}
\end{array}\right) .
$$

In addition, we also have

$$
\Upsilon_{n}=\left(\begin{array}{cc}
\sigma_{n}-\varsigma_{n}-\varsigma_{n-1} & -\varsigma_{n} \\
\varsigma_{n} & \sigma_{n}+\varsigma_{n}+\varsigma_{n-1}
\end{array}\right),
$$

with

$$
\begin{aligned}
\rho_{n} & =\sum_{l=0}^{\lfloor 3 n / 4\rfloor} \sum_{m=0}^{l}(-1)^{n-l} C_{n-l}^{m} C_{2(n-l)}^{l-m} 2^{n-l-m} T_{2(n-l)}, \\
\chi_{n} & =\sum_{l=0}^{\lfloor(3 n+1) / 4\rfloor} \sum_{m=0}^{l}(-1)^{n-l} C_{n-l}^{m} C_{2(n-l)+1}^{l-m} 2^{n-l-m} T_{2(n-l)+1}, \\
\sigma_{n} & =\sum_{l=0}^{\lfloor 5 n / 6\rfloor} \sum_{m=0}^{l} C_{n-l}^{m} C_{4(n-l)}^{l-m} 2^{n-l-m} H_{2(n-l)}, \\
\varsigma_{n} & =\sum_{l=0}^{\lfloor(5 n+2) / 6\rfloor} \sum_{m=0}^{l} C_{n-l}^{m} C_{4(n-l)+2}^{l-m} 2^{n-l-m} H_{2(n-l)+1} .
\end{aligned}
$$

Here we denote $T_{m}=\frac{t^{m}}{m !}, \quad H_{m}=\frac{(4 \alpha t)^{m}}{m !}$.

Moreover, if the arbitrary complex constant vector $Z$ has the following form

$$
Z=\sum_{j=1}^{\infty} Z_{j} \delta^{j}
$$


with $Z_{j}$ 's are free complex constant vectors, then solution (7) has an expansion

$$
\begin{aligned}
& \left.\Psi\right|_{\lambda=\mathrm{i}(1+\delta)}=\sum_{n=0}^{\infty} \Psi_{n} \delta^{n}, \\
& \Psi_{n}=\left(\begin{array}{c}
\psi_{n} \\
\phi_{n}
\end{array}\right)=e^{\mathrm{i} t} A \sum_{k=0}^{n} \sum_{j=0}^{n} F_{k} G_{j} Z_{n-k-j} .
\end{aligned}
$$

Furthermore, on using the binomial theorem,

$$
\begin{aligned}
& \mathrm{i}^{j}(1+\delta)^{j} \psi(\delta)=\psi[j, 0]+\psi[j, 1] \delta+\cdots+\psi[j, N] \delta^{N}+\cdots, \\
& \mathrm{i}^{j}(1+\delta)^{j} \phi(\delta)=\phi[j, 0]+\phi[j, 1] \delta+\cdots+\phi[j, N] \delta^{N}+\cdots
\end{aligned}
$$

we obtain

$$
\psi[j, n]=\sum_{k=0}^{n} \mathrm{i}^{j} C_{j}^{n-k} \psi_{k}, \quad \phi[j, n]=\sum_{k=0}^{n} \mathrm{i}^{j} C_{j}^{n-k} \phi_{k} .
$$

Now, by means of a generalized Darboux transformation introduced by Guo et al [7], it follows directly that the $N$ th-order rogue wave solutions for Hirota equation as

$$
u[N]=\left[1-2 \mathrm{i} \frac{D_{N 2}}{D_{N 1}}\right] e^{\mathrm{i} t},
$$

where

$$
\begin{aligned}
& D_{N 1}=\left|\begin{array}{cccccc}
\phi[N-1,0] & \cdots & \phi[N-1, N-1] & -\psi[N-1,0]^{*} & \cdots & -\psi[N-1, N-1]^{*} \\
\ldots & \cdots & \cdots & \cdots & \cdots & \cdots \\
\phi[0,0] & \cdots & \phi[0, N-1] & -\psi[0,0]^{*} & \cdots & -\psi[0, N-1]^{*} \\
\psi[N-1,0] & \cdots & \psi[N-1, N-1] & \phi[N-1,0]^{*} & \cdots & \phi[N-1, N-1]^{*} \\
\ldots & \cdots & \cdots & \cdots & \cdots & \cdots \\
\psi[0,0] & \cdots & \psi[0, N-1] & \phi[0,0]^{*} & \cdots & \phi[0, N-1]^{*}
\end{array}\right|, \\
& D_{N 2}=\left|\begin{array}{cccccc}
\psi[N, 0] & \cdots & \psi[N, N-1] & \phi[N, 0]^{*} & \cdots & \phi[N, N-1]^{*} \\
\phi[N-2,0] & \cdots & \phi[N-2, N-1] & -\psi[N-2,0]^{*} & \cdots & -\psi[N-2, N-1]^{*} \\
\cdots & \cdots & \cdots & \cdots & \cdots & \cdots \\
\phi[0,0] & \cdots & \phi[0, N-1] & -\psi[0,0]^{*} & \cdots & -\psi[0, N-1]^{*} \\
\psi[N-1,0] & \cdots & \psi[N-1, N-1] & \phi[N-1,0]^{*} & \cdots & \phi[N-1, N-1]^{*} \\
\cdots & \cdots & \cdots & \cdots & \cdots & \cdots \\
\psi[0,0] & \cdots & \psi[0, N-1] & \phi[0,0]^{*} & \cdots & \phi[0, N-1]^{*}
\end{array}\right| .
\end{aligned}
$$


Thus, the explicit formula of $N$ th order rogue waves of Hirota is treated without any derivatives computation, which is different from the results obtained by Guo et al [7].

\section{Illustrative examples}

To illustrate the mechanism of deriving rogue waves using formulations developed in the previous section, we shall work out the analytical expressions for the two lowest order rogue waves explicitly. The higher order modes have also been deduced. Precise expressions will not be tabulated as their forms are rather lengthy. In terms of the wave profiles, we shall plot directly the third order rogue waves, as diagrams for the two lowest order rogue waves have been given earlier in the literature [19]. Firstly, we set $N=1$ in (21). In this case,

$$
\begin{aligned}
& F_{0}=\left(\begin{array}{cc}
1+x & x \\
-x & 1-x
\end{array}\right), \quad Z_{0}=\left(\begin{array}{l}
z_{01} \\
z_{02}
\end{array}\right), \\
& G_{0}=\left(\begin{array}{cc}
1-6 \alpha t+\mathrm{i} t & -6 \alpha t+\mathrm{i} t \\
6 \alpha t-\mathrm{i} t & 1+6 \alpha t-\mathrm{i} t
\end{array}\right),
\end{aligned}
$$

and

$$
\begin{aligned}
& \Psi_{0}=\left(\begin{array}{c}
\psi_{0} \\
\phi_{0}
\end{array}\right)=\exp (\mathrm{i} t / 2) A F_{0} G_{0} Z_{0}, \\
& \phi[0,0]=\phi_{0}, \quad \psi[0,0]=\psi_{0}, \\
& \phi[1,0]=\mathrm{i} \phi_{0}, \quad \psi[1,0]=\mathrm{i} \psi_{0},
\end{aligned}
$$

More precisely, the lowest order rogue wave is

$$
u[1]=\left[1-2 \mathrm{i} \frac{D_{12}}{D_{11}}\right] e^{\mathrm{i} t},
$$

where

$$
D_{11}=\left|\begin{array}{cc}
\phi_{0} & -\psi_{0}^{*} \\
\psi_{0} & \phi_{0}^{*}
\end{array}\right|, \quad D_{12}=\left|\begin{array}{cc}
\mathrm{i} \psi_{0} & -\mathrm{i} \phi_{0}^{*} \\
\psi_{0} & \phi_{0}^{*}
\end{array}\right| .
$$

The explicit formula of (22) reads

$$
u[1]=-1-2 \frac{(1+2 i t)\left(z_{01}+z_{02}\right)^{2}}{\triangle},
$$


where

$$
\begin{aligned}
\triangle=\quad & \left(72 \alpha^{2} z_{01}^{2}+144 \alpha^{2} z_{01} z_{02}+72 \alpha^{2} z_{02}^{2}+2 z_{01}^{2}+4 z_{01} z_{02}+2 z_{02}^{2}\right) t^{2} \\
& +\left(\left(-24 \alpha z_{01}^{2}-48 \alpha z_{01} z_{02}-24 \alpha z_{02}^{2}\right) x-12 \alpha z_{01}^{2}+12 \alpha z 02^{2}\right) t \\
& +\left(2 z_{01}^{2}+4 z_{01} z_{02}+2 z_{02}^{2}\right) x^{2}+\left(2 z_{01}^{2}-2 z_{02}^{2}\right) x+z_{01}^{2}+z_{02}^{2}
\end{aligned}
$$

When $z_{01}=z_{02}$, it can be reduced to

$$
u[1]=-1+4 \frac{1+2 i t}{1+4(x-6 \alpha t)^{2}+4 t^{2}}
$$

which is the obtained formula with $\alpha=-\alpha_{3}$ and interchanging $x, t$ in [19].

To obtain the second-order rogue waves, we take $N=2$. In this case, the formulation simplifies to

$$
F_{1}=\left(\begin{array}{cc}
x+x^{2}+\frac{1}{3} x^{3} & \frac{1}{3} x^{3} \\
-\frac{1}{3} x^{3} & -x+x^{2}-\frac{1}{3} x^{3}
\end{array}\right),
$$

and

$$
\begin{aligned}
& \Theta_{0}=\left.F_{0}\right|_{x=-2 \alpha t}, \quad \Theta_{1}=\left.F_{1}\right|_{x=-2 \alpha t} \\
& \Pi_{0}=\left(\begin{array}{cc}
1+\mathrm{i} t & \mathrm{i} t \\
-\mathrm{i} t & 1-\mathrm{i} t
\end{array}\right), \\
& \Pi_{1}=\left(\begin{array}{cc}
2 \mathrm{i} t-t^{2}-\frac{1}{3} i t^{3} & -\frac{1}{3} \mathrm{i} t^{3}+2 i t \\
\frac{1}{3} \mathrm{i} t^{3}-2 \mathrm{i} t & -2 \mathrm{i} t-t^{2}+\frac{1}{3} \mathrm{i} t^{3}
\end{array}\right), \\
& \Upsilon_{0}=\left(\begin{array}{cc}
1-4 \alpha t & -4 \alpha t \\
4 \alpha t & 1+4 \alpha t
\end{array}\right) \\
& \Upsilon_{1}=\left(\begin{array}{cc}
32 \alpha^{2} t^{2}-\frac{32}{3} \alpha^{3} t^{3}-12 \alpha t & -\frac{32}{3} \alpha^{3} t^{3}-8 \alpha t \\
\frac{32}{3} \alpha^{3} t^{3}+8 \alpha t & 32 \alpha^{2} t^{2}+\frac{32}{3} \alpha^{3} t^{3}+12 \alpha t
\end{array}\right), \\
& G_{1}=\Theta_{0} \Pi_{0} \Upsilon_{1}+\Theta_{0} \Pi_{1} \Upsilon_{0}+\Theta_{1} \Pi_{0} \Upsilon_{1},
\end{aligned}
$$

and thus $\Psi_{1}$ can be calculated as

$$
\begin{aligned}
& \Psi_{1}=\left(\begin{array}{c}
\psi_{1} \\
\phi_{1}
\end{array}\right)=\exp (-i t / 2) A\left[\left(F_{1} G_{0}+F_{0} G_{1}\right) Z_{0}+F_{0} G_{0} Z_{1}\right], \\
& \phi[0,1]=\phi_{1}, \psi[0,1]=\psi_{1}, \phi[2,0]=-\phi_{0}, \psi[2,0]=-\psi_{0}, \\
& \phi[1,1]=\mathrm{i}\left(\phi_{0}+\phi_{1}\right), \quad \psi[1,1]=\mathrm{i}\left(\psi_{0}+\psi_{1}\right), \\
& \phi[2,1]=-\left(2 \phi_{0}+\phi_{1}\right), \quad \psi[2,1]=-\left(2 \psi_{0}+\psi_{1}\right) .
\end{aligned}
$$


Consequently, the second-order rogue wave is

$$
u[2]=\left[1-2 \mathrm{i} \frac{D_{22}}{D_{21}}\right] e^{\mathrm{i} t},
$$

where

$$
\begin{gathered}
D_{21}=\left|\begin{array}{llll}
\phi[1,0] & \phi[1,1] & -\psi[1,0]^{*} & -\psi[1,1]^{*} \\
\phi[0,0] & \phi[0,1] & -\psi[0,0]^{*} & -\psi[0,1]^{*} \\
\psi[1,0] & \psi[1,1] & -\phi[1,0]^{*} & -\phi[1,1]^{*} \\
\psi[0,0] & \psi[0,1] & -\phi[0,0]^{*} & -\phi[0,1]^{*}
\end{array}\right|, \\
D_{22}=\left|\begin{array}{llll}
\psi[2,0] & \psi[2,1] & -\phi[2,0]^{*} & -\phi[2,1]^{*} \\
\phi[0,0] & \phi[0,1] & -\psi[0,0]^{*} & -\psi[0,1]^{*} \\
\psi[1,0] & \psi[1,1] & -\phi[1,0]^{*} & -\phi[1,1]^{*} \\
\psi[0,0] & \psi[0,1] & -\phi[0,0]^{*} & -\phi[0,1]^{*}
\end{array}\right| .
\end{gathered}
$$

Again, the rogue wave is obtained without any derivative calculations.

The explicit formula of third order rogue waves is too long to write here. It is interesting to illustrate that the features of Figs 1-2 depict some interesting asymmetrical third order rogue waves with $Z_{0}=(1,0)^{T}$ and $Z_{1}=Z_{2}=(0,0)^{T}$ and Figs 3-4 display they form a half circle texture with $Z_{0}=(1,0)^{T}, Z_{1}=(1,5)^{T}$ and $Z_{2}=(0,0)^{T}$ by exploiting our results.

\section{Computational study of stability}

Although there have been extensive studies $[15,16,18]$ on the derivation of the lowest order and higher order rogue waves, their stability properties have received comparatively little attention. The stability of rogue waves can be studied by a variety of theoretical and computational techniques. Analytical approaches, e.g. finding the eigenvalues of the linearized differential operators and setting upper bounds by examining integrals of energy, may involve excessive details far more than adequate for the present consideration. Furthermore, it is physically more relevant to study the evolution of a perturbed profile from an experimental or practical point of view. In fact the robustness for the emergence of rogue waves has been investigated along this line of reasoning for many rogue wave problems, e.g. watch-hand-like modes in three-wave interactions [21], metamaterials [22], and long-wave-short-wave 

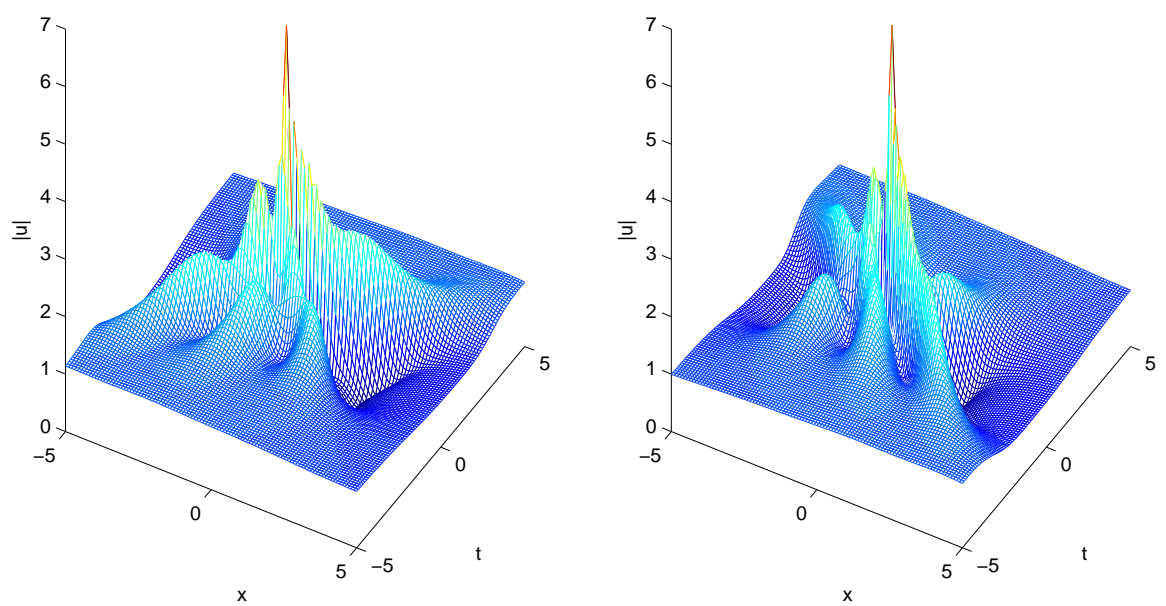

Figure 1: (Color online) Amplitude of the third order rogue waves versus $x$ and $t$ with parameters $Z_{0}=(1,0)^{T}$ and $Z_{1}=Z_{2}=(0,0)^{T}$ corresponding to $\alpha=1 / 15$ (left) and $\alpha=-1 / 15$ (right). There is one single dominant peak. 

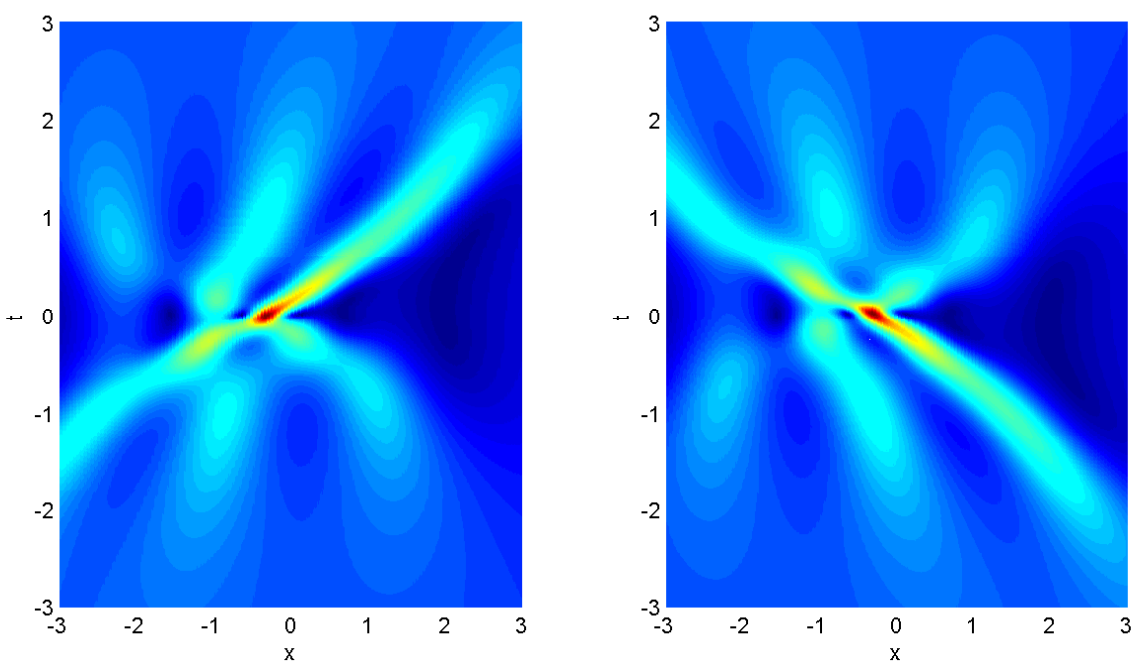

Figure 2: (Color online) Contour (planar) plot of the third order rogue wave with parameters given as those in Figure 1.
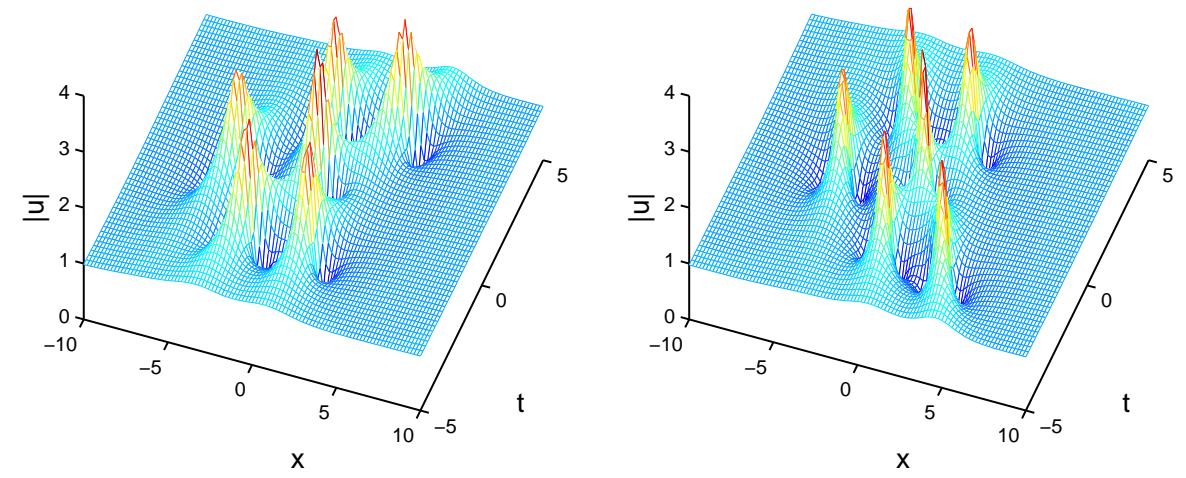

Figure 3: (Color online) Amplitude of the third order rogue waves versus $x$ and $t$ with parameters $Z_{0}=(1,0)^{T}, Z_{1}=(1,5)^{T}$ and $Z_{2}=(0,0)^{T}$ corresponding to $\alpha=1 / 15$ (left) and $\alpha=-1 / 15$ (right). The peaks are more or less of the same magnitude. 

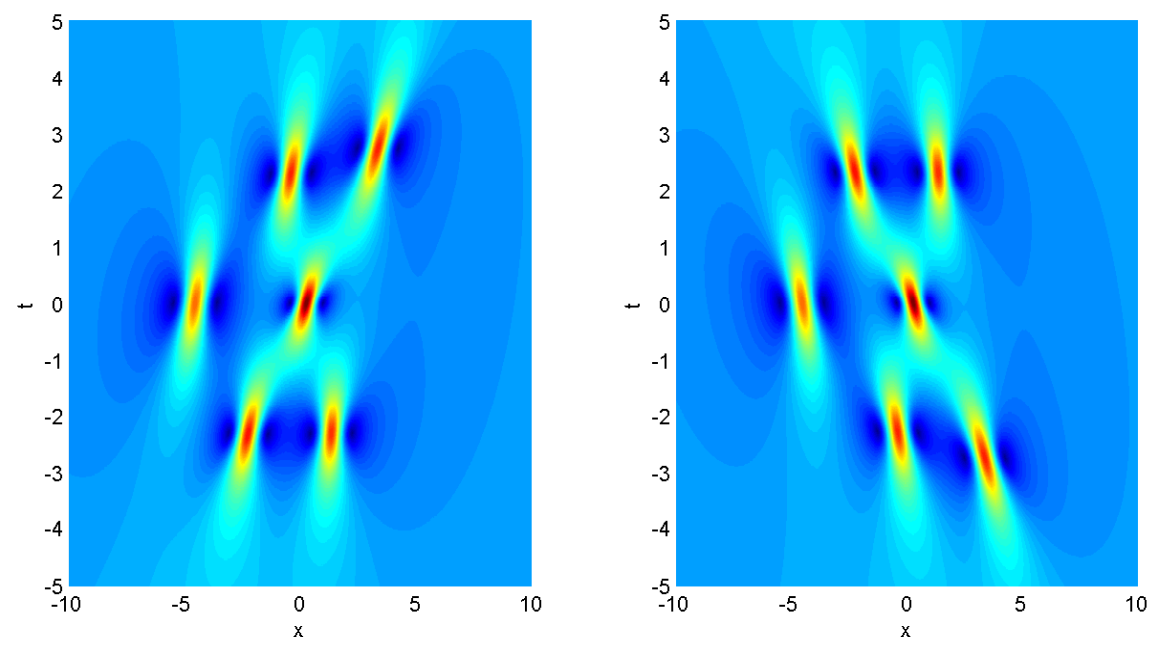

Figure 4: (Color online) Contour (planar) plot of the third order rogue wave with parameters given as those in Figure 3.

resonance [23]. For classical and intensively studied models, e.g. the Peregrine breather rogue wave of the nonlinear Schrödinger equation (NLSE), agreements are obtained between numerical simulations and theoretical approaches like eigenvalues consideration [24, 25, 26]. Given the similarity between the Hirota equation and the NLSE, it is highly likely that a similar scenario will prevail here. Stability is thus addressed by numerical simulations of exact wave profiles perturbed by a small amplitude random noise. Rogue waves having their shapes significantly distorted or allowing dramatic growth of any background noise to a magnitude comparable with the rogue wave itself will be termed unstable. A more elaborate approach, namely, on studying the eigenvalue spectrum of the linearized evolution equation, will be left for future efforts.

The numerical solution to (2) is obtained by employing a fourth-order centered finite difference scheme to evaluate the $x$ derivatives and the fourth order Runge-Kutta scheme to evaluate the $t$ derivative.

The domain $-\frac{L}{2} \leq x<\frac{L}{2}$ is discretized into $N$ equally spaced intervals. At any step $x_{p}$ and step $t_{q}$, the numerical solution to $(2)$ is given by $u(x, t)=$ $u_{q}^{p}$.

The fourth-order centered finite difference scheme is based on the Taylor expansion of $u(x, t)$ : 


$$
\begin{aligned}
u_{x x x} & =\frac{-u_{q}^{p+3}+8 u_{q}^{p+2}-13 u_{q}^{p+1}+13 u_{q}^{p-1}-8 u_{q}^{p-2}+u_{q}^{p-3}}{8(\Delta x)^{3}}, \\
u_{x x} & =\frac{-u_{q}^{p+2}+16 u_{q}^{p+1}-30 u_{q}^{p}+16 u_{q}^{p-1}-u_{q}^{p-2}}{12(\Delta x)^{2}} \\
u_{x} & =\frac{-u_{q}^{p+2}+8 u_{q}^{p+1}-8 u_{q}^{p-1}+u_{q}^{p-2}}{12 \Delta x}
\end{aligned}
$$

where $\Delta x$ is the step size.

As usual for boundary value problems, calculations of the higher order derivatives near the boundary incur the definitions of grid points beyond the nominal boundaries. Here a periodic boundary condition is invoked. Generalizing this for all the difference schemes used, we have $x_{-k}=x_{N-k+1}$, where $k$ is a positive number and $N$ is the number of steps in $x$.

In this section, we are interested in exploring the growth phase of the rogue wave. Here we only consider the first and second order rogue waves. A wave with amplitude $20 \%$ to $30 \%$ larger than the background is chosen as the initial condition. According to the analytical solutions of both rogue waves, the maximum amplitudes occur at $t=0$. As a result, negative initial values of $t$ were chosen in order to simulate the growth phase of the rogue wave.

For the lowest order rogue wave, we employ the following initial condition:

$$
u\left(x, t_{0}\right)=-\left[1-\frac{1+2 \mathrm{i} t_{0}}{\left(x-6 \alpha t_{0}+1 / 2\right)^{2}+t_{0}^{2}+1 / 4}\right] e^{\mathrm{i} t_{0}},
$$

where $t_{0}=-3$.

For the second order rogue wave, we employ the following initial condition:

$$
u\left(x, t_{0}\right)=\left[1+\frac{G_{2}+\mathrm{i} t_{0} H_{2}}{D_{2}}\right] e^{\mathrm{i} t_{0}},
$$


where

$$
\begin{aligned}
G_{2}= & 12\left(-16 x^{4}-384 \alpha t_{0} x^{3}-24\left(4\left(36 \alpha^{2}+1\right) t_{0}^{2}+1\right) x^{2}-\right. \\
& 96 \alpha t_{0}\left(12\left(12 \alpha^{2}+1\right) t_{0}^{2}+7\right) x-16\left(1296 \alpha^{4}+216 \alpha^{2}+5\right) t_{0}^{4} \\
& \left.-72\left(44 \alpha^{2}+1\right) t_{0}^{2}+3\right), \\
H_{2}= & 24\left(-16 x^{4}-384 \alpha t_{0} x^{3}-8\left(\left(432 \alpha^{2}+4\right) t_{0}^{2}-3\right) x^{2}-\right. \\
& \left.96 \alpha t_{0} x\left(4\left(36 \alpha^{2}+1\right) t_{0}^{2}+1\right)-16\left(36 \alpha^{2}+1\right)^{2} t_{0}^{4}-8\left(180 \alpha^{2}+1\right) t_{0}^{2}+15\right), \\
D_{2}= & 64 x^{6}+2304 \alpha t_{0} x^{5}-432\left(624 \alpha^{4}-40 \alpha^{2}-1\right) t_{0}^{4}+36\left(556 \alpha^{2}+11\right) t_{0}^{2}+9 \\
& +64\left(36 \alpha^{2}+1\right)^{3} t_{0}^{6}+384 \alpha x^{3}\left(12\left(60 \alpha^{2}+1\right) t_{0}^{2}-1\right) t_{0}+48 x^{4}\left(\left(720 \alpha^{2}+4\right) t_{0}^{2}\right. \\
& +1)+12\left(16\left(6480 \alpha^{4}+216 \alpha^{2}+1\right) t_{0}^{4}-24\left(60 \alpha^{2}+1\right) t_{0}^{2}+9\right) x^{2} \\
& +144 \alpha t_{0}\left(16\left(36 \alpha^{2}+1\right)^{2} t_{0}^{4}+\left(8-864 \alpha^{2}\right) t_{0}^{2}+17\right) x, \\
t_{0}= & -5 .
\end{aligned}
$$

In order to test the stability of the rogue wave solutions, we consider numerically the evolution of both the exact solution and the exact solution coupled with a $5 \%$ perturbation. In addition, we consider (i) phase, (ii) amplitude, (iii) localized and (iv) non-localized perturbations to the exact solution. The resulting initial conditions have the forms:

- (i) exact solution $+\epsilon \cos [2 \pi(x+\phi) / L]$

- (ii) exact solution $+\epsilon r \cos [2 \pi x / L]$

- (iii) exact solution $+\epsilon \exp \left[-\left(x^{2}\right)\right]$

- (iv) exact solution $+\epsilon \exp \left[-(x-5)^{2}\right]+\epsilon \exp \left[-(x+5)^{2}\right]$,

where $\phi$ is a random function bounded in $[-1,1]$ and $r$ is a random function bounded in $[0,1]$. These random functions were obtained using a standard MATLAB random seed generator.

The Hirota equation, just like the nonlinear Schrödinger equation, exhibits both regimes of modulation instability and modulation stability. The present situation, with cubic nonlinear term and dispersive terms of the same sign, will display instability. Hence, for the case of zero noise (top panel of Figure 5), even though the first order rogue wave can clearly emerge, we can see that eventually instabilities of the background will start to appear due to numerical round off errors. For the case with five percent noise (bottom 

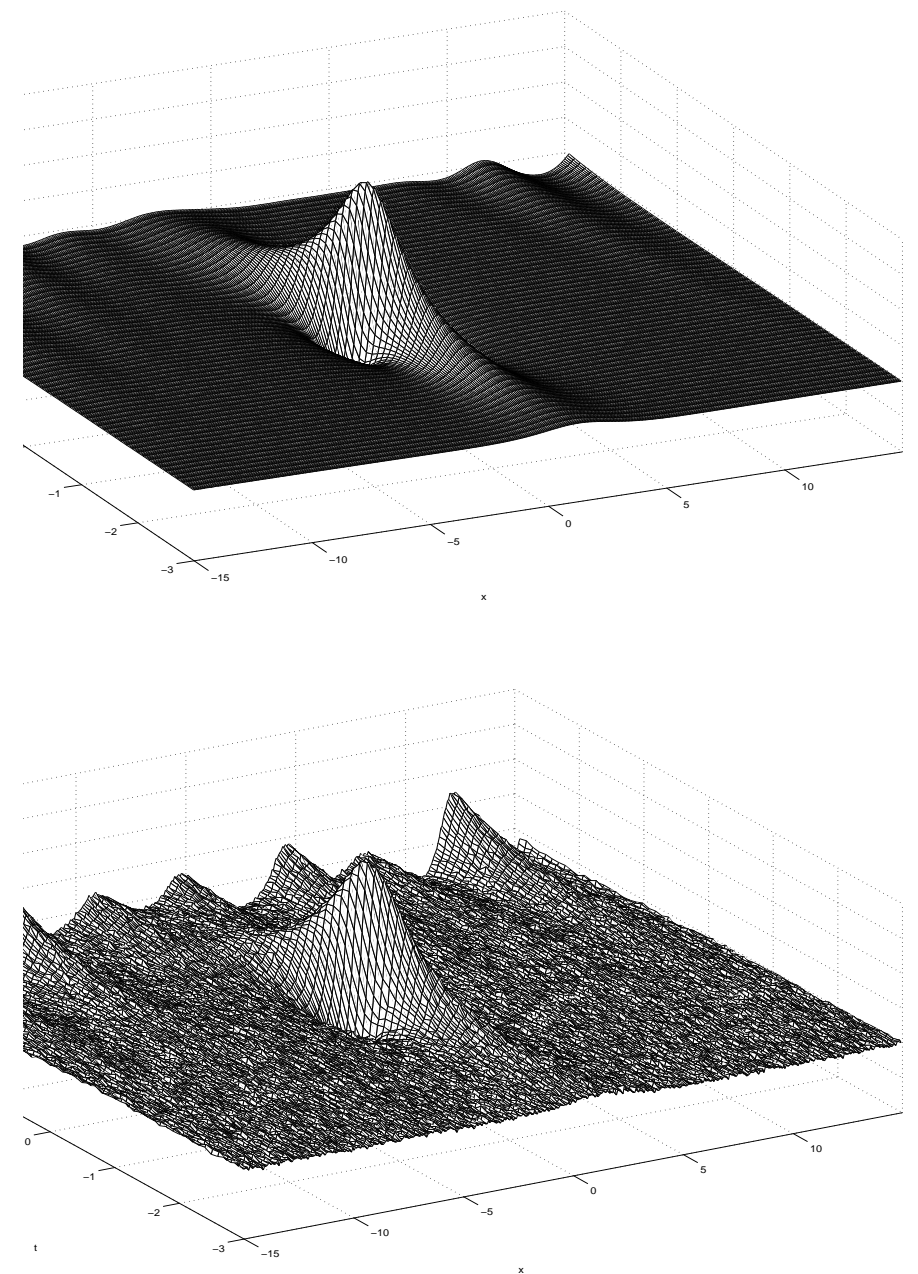

Figure 5: Top panel: Evolution of the first order rogue wave with initial conditions given by Equation (27) (top panel) and with $5 \%$ noise perturbation (bottom panel) using $t_{0}=-3$ and $\alpha=-0.1$. 


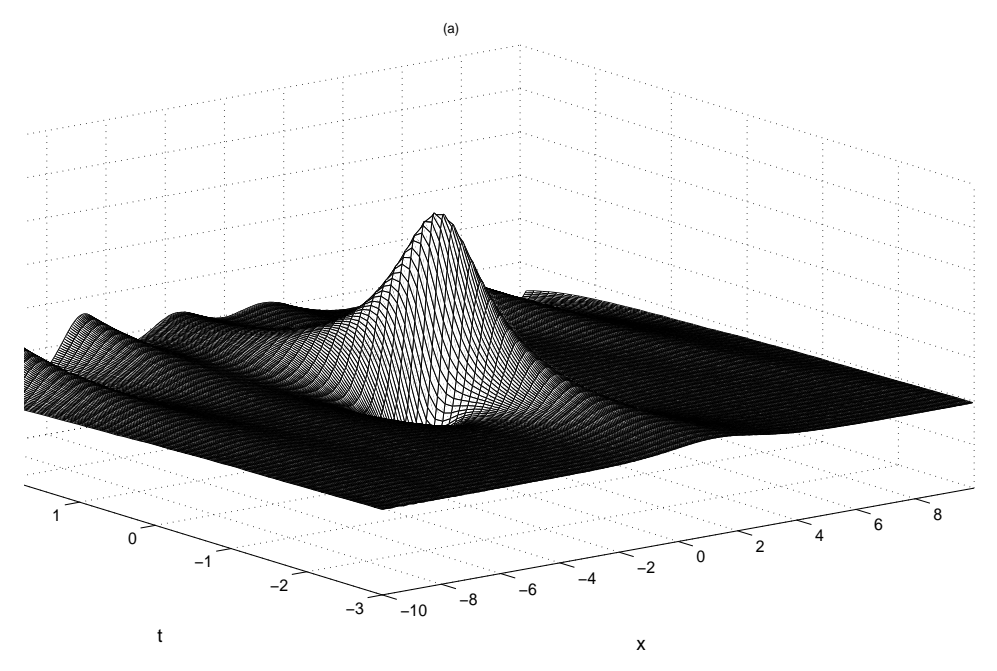

(b)

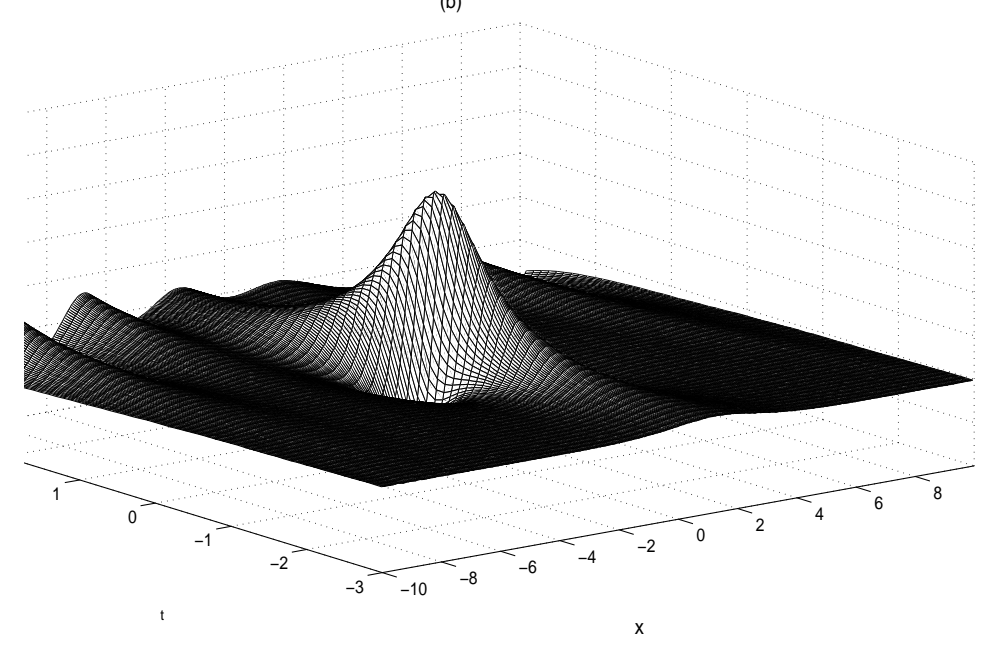



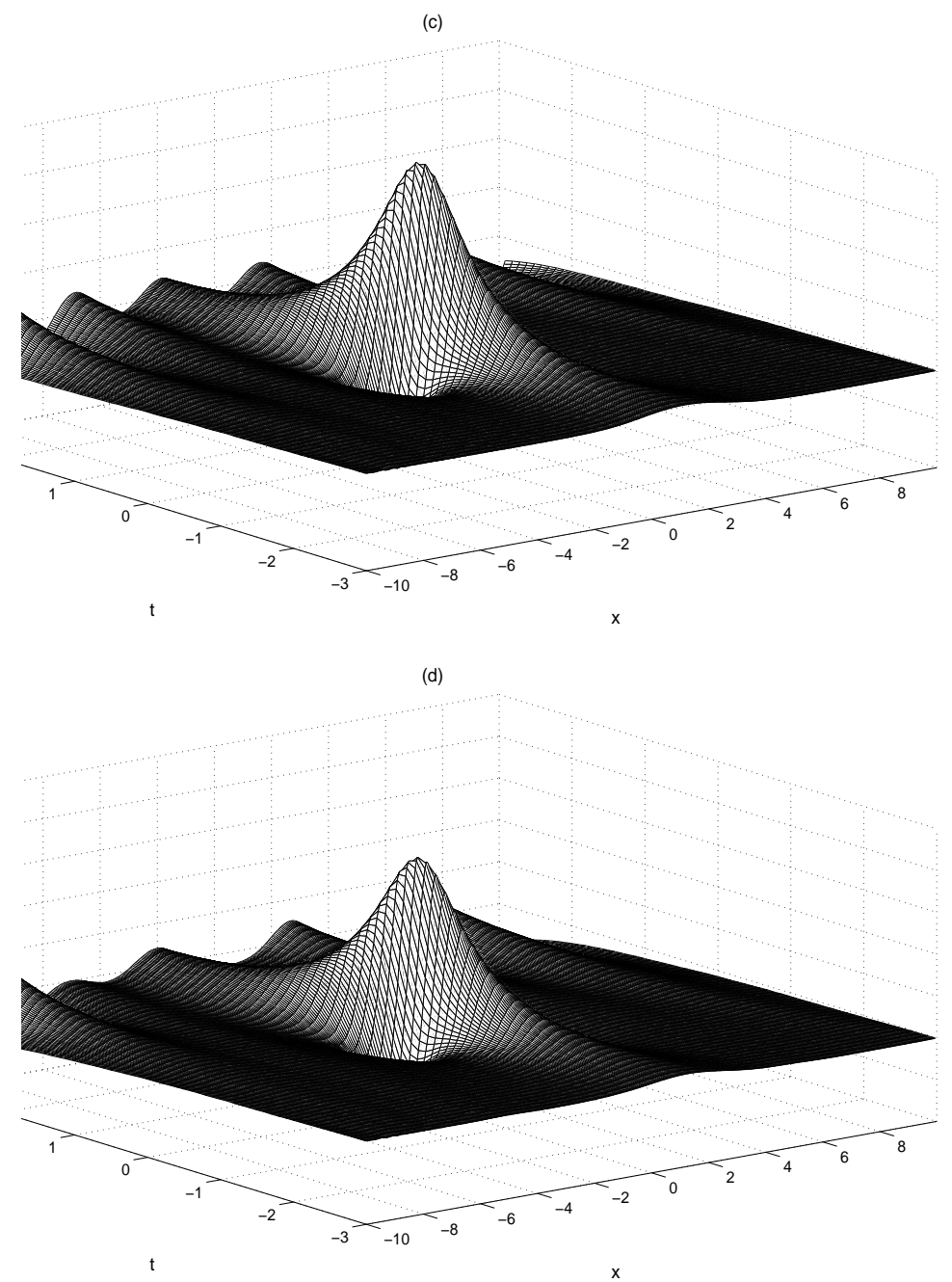

Figure 6: Evolution of the first order rogue wave with initial conditions given by (27) with (a) phase perturbation, (b) amplitude perturbation, (c) localized perturbation and (d) non-localized perturbation using $\epsilon=0.01, t_{0}=-3$ and $\alpha=-0.1$. 


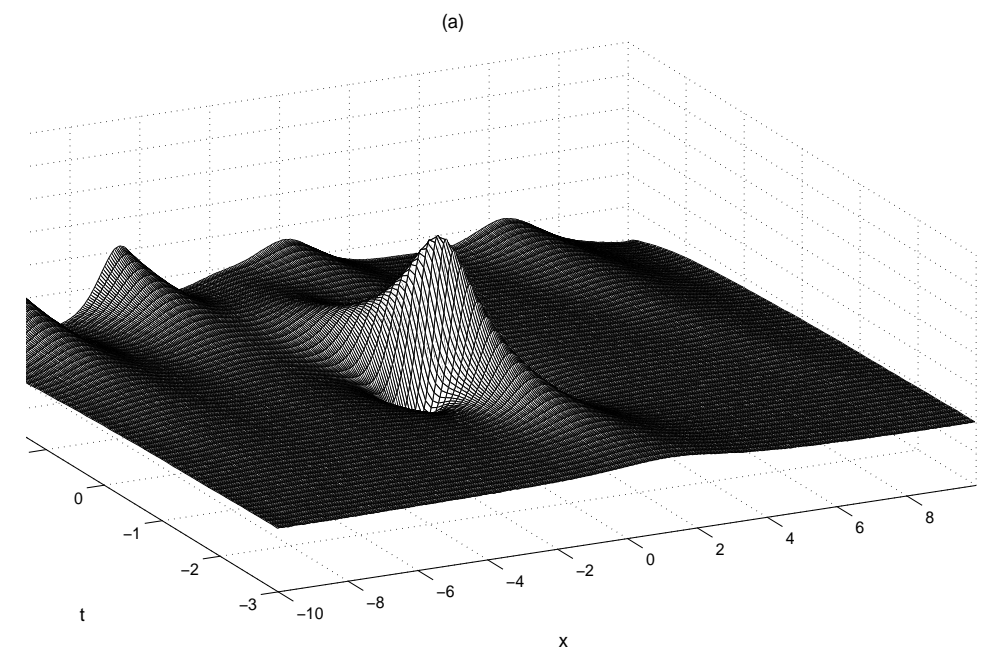

(b)

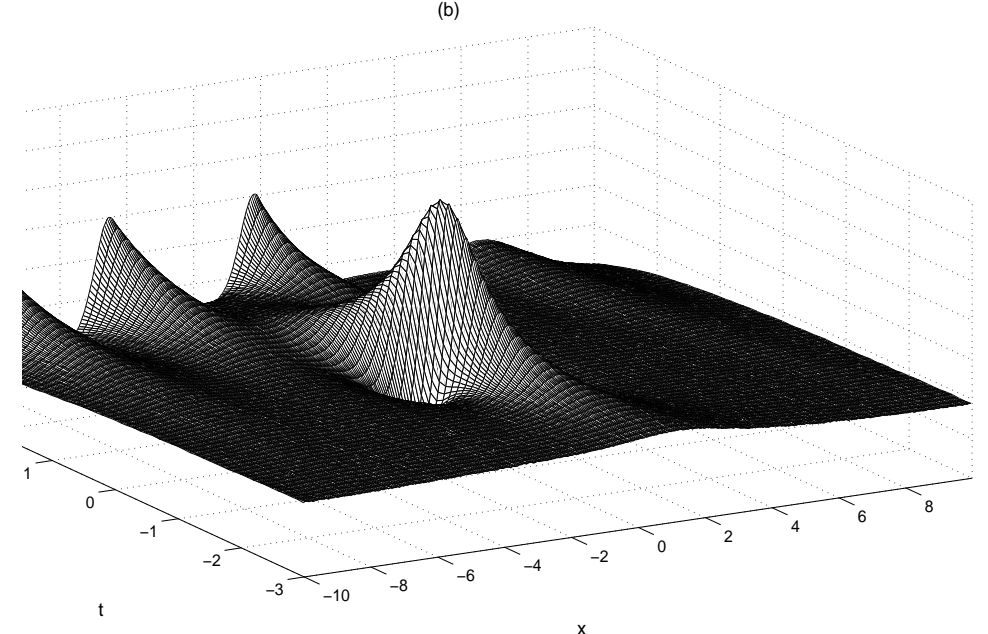

Figure 7: Evolution of the first order rogue wave with initial conditions given by (27) with phase perturbation using $t_{0}=-3, \alpha=-0.1$, (a) $\epsilon=0.03$ and (b) $\epsilon=0.05$. 



Figure 8: Evolution of the second order rogue wave with initial conditions given by (28) with (a) phase perturbation and (b) amplitude perturbation using $\epsilon=0.01, t_{0}=-3$ and $\alpha=-0.1$. 
panel of Figure 5), the rogue wave still remains more or less intact, even though the background instabilities are observed to be stronger.

In Figure 6, we consider the phase, amplitude, localized and non-localized perturbations to the first order rogue wave for noise of $1 \%$ (i.e. $\epsilon=0.01$ ). We clearly see that there is no significant difference among the four different types of perturbations and that the first order rogue wave is still evident. When the noise is increased to $3 \%$ and $5 \%$ (i.e. $\epsilon=0.03$ and 0.05 ), we observe that the first order rogue wave can still emerge (Figure 7) though background instabilities are observed to be stronger with increasing noise. Hence, it is reasonable to conclude the first order rogue wave probably possesses reasonably robust structural stability.

For the second order rogue wave, we can generally see that the background noise increases so rapidly that the rogue wave is eventually masked. The background instabilities evolve independently alongside the second order mode. This is evident when we compare between the first and second order rogue wave cases for $\epsilon=0.01$ (ref. Figures $6(\mathrm{a}-\mathrm{b})$ and $8(\mathrm{a}-\mathrm{b})$ ). However, it would seem that there is a slight difference between the evolution of the second order rogue wave for initial conditions with phase and amplitude perturbations. The background noise grows more slowly for amplitude perturbations (ref. Figure $8(\mathrm{~b})$ ) than that for the phase perturbations (ref. Figure $8(\mathrm{a}))$ ). Hence the numerical evidence strongly suggests that the second order rogue wave will be less stable than the lowest order rogue wave. Similar works for the third and higher order rogue waves will be left for future studies.

\section{Conclusion}

Rogue waves have attracted intensive attention as they arise frequently in the numerical simulations of the nonlinear Schrödinger equation and other well studied models [27, 28], and can be observed experimentally [5, 29]. It is thus worthwhile to conduct a penetrating study on the various theoretical techniques in the analysis of evolution equations from the nonlinear Schrödinger family. The Hirota equation is a member of this family, and incorporates third order dispersion and a nonlinearity resembling 'selfsteepening' effects [14] in optical fibers.

In this work we utilize our approach on a separation of variable algorithm proposed in the reference [1] to solve the Lax pair associated with 
the Hirota equation. The subsequent Taylor series expansion permits an explicit procedure to construct the higher order rogue waves. More precisely, the eigenfunctions of earlier works require the $n$-th derivative of intermediate functions which are difficult or impossible to construct or to represent explicitly. We surmount these difficulties by using an exponential function representation.

However, queries and challenges remain. We have verified that known first and second order rogue waves from the literature can be recovered by our procedure, by choosing special starting conditions for the algorithm outlined in Sections 3, 4. Whether further solutions can be obtained by choosing arbitrary initial conditions must await future efforts.

Although analytical formulas of rogue waves have been given extensively in the literature for various nonlinear evolution equations, computational studies of their stability have been given comparatively little attention. Here the stability of the first and second order rogue waves of the Hirota equation was studied numerically, by tracing the evolution of an exact solution perturbed by random noise.

Since the basic state belongs to the regime of modulation instability, eventually small disturbance of the background plane wave will grow. However, the rogue wave can still emerge intact in the absence of any perturbation on the background. In the presence of a five percent noise, the existence and dynamics of the lowest order rogue wave are more or less unaffected, even though the modulation instability may appear soon after the rogue wave subsides. In contrast, in corresponding simulations of the second order rogue wave, many peaks, some completely unrelated to the rogue wave mode, were observed, suggesting that this mode is less structurally stable.

Theoretically there are also many deeper issues. An important one is the large time behavior of modulation instability. For the nonlinear Schrödinger equation, the answer for this question already involves intriguing analysis, e.g. properties of higher order breathers and dynamics of homoclinic orbits $[23,24]$. A similar analysis incorporating the third order derivative must await future efforts. On the aspect of further extension, a great theoretical challenge is to apply this separation of variable technique to other nonlinear evolution equations, e.g. the derivative nonlinear Schrödinger equations [32], and envelope system coupled to a real field (e.g. the induced mean flow in fluid mechanics) [33, 34]. 


\section{Acknowledgement}

The work was supported in part by the National Natural Science Foundation of China (No. 11571079), The Natural Science Foundation of Shanghai (No. 14ZR1403500), Shanghai Pujiang Program (No. 14PJD007) and the Young Teachers Foundation (No. 1411018) of Fudan University and Yunnan province project Education Fund (No. 2013C012). Partial support for KWC has been provided by the Research Grants Council contract HKU711713E. Furthermore, the authors are very grateful to Professor Peter D. Miller, Professor John E. Fornaess and Professor Zhengde Dai for their enthusiastic support and useful suggestions.

\section{References}

[1] G. Mu, Z. Y. Qin, J. Phys. Soc. Jpn. 83 (2014) 104001

[2] K. Dysthe, H. E. Krogstad, P. Miller, Annu. Rev. Fluid Mech. 40 (2008) 287.

[3] N. Akhmediev, E. Pelinovsky, Eur. Phys. J. Special Topics 185 (2010) $1-4$.

[4] I. Didenkulova, E. Pelinovsky, Nonlinearity 24 (2011) R1

[5] L. H. Ying, Z. Zhuang, E. J. Heller, L. Kaplan, Nonlinearity 24 (2011) R67.

[6] S. K. Sharma, H. Bailung, J. Geophys. Res. Space Phys. 118 (2013) 919-924

[7] D. R. Solli, C. Ropers, P. Koonath, B. Jalali, Nature (London) 450 (2007) 1054C1057

[8] W. M. Moslem, P. K. Shukla, B. Eliasson, EPL 96 (2011) 25002

[9] Y. V. Bludov, V. V. Konotop, N. Akhmediev, Phys. Rev. A 80 (2009) 033610

[10] L. C. Zhao, Ann. Phys. 329 (2013) 73-79 
[11] M. Onorato, S. Residori, U. Bortolozzo, A. Montina, F. T. Arecchi, Phys. Rep. 528 (2013) 47-89

[12] A. D. D. Craik, Wave Interactions and Fluid Flows, Cambridge University Press, New York, 1985

[13] Y. S. Kivshar, G. P. Agrawal, Optical Solitons: From Optical Fibers to Photonic Crystals, Academic Press, San Diego, 2003

[14] D. H. Peregrine, J. Aust. Math. Soc., Ser. B 25 (1983) 16-43

[15] N. Akhmediev, A. Ankiewicz, J. M. Soto-Crespo, Phys. Rev. E 80 (2009) 026601

[16] Y. Ohta and J. Yang, Proc. R. Soc. A 468 (2012) 1716-1740

[17] P. Dubard, P. Gaillard, C. Kleina, V. B. Matveev, Eur. Phys. J. Special Topics 185 (2010) 247-258

[18] J. S. He, H. R. Zhang, L. H. Wang, K. Porsezian, A. S. Fokas, Phys. Rev. E 87 (2013) 052914

[19] A. Ankiewicz, J. M. Soto-Crespo, N. Akhmediev, Phys. Rev. E 81 (2010) 046602

[20] L. J. Li, Z. W. Wu, L.h. Wang, J. S. He, Annals of Physics, 334 (2013) $198-211$

[21] S. Chen, J. M. Soto-Crespo, P. Grelu, Opt. Expr. 23 (2015) 349

[22] B. G. O. Essama, J. Atangana, F. Biya-Motto, B. Mokhtari, N. C. Eddeqaqi, T. C. Kofane, Opt. Commun. 331 (2014) 334

[23] S. Chen, J. M. Soto-Crespo, P. Grelu, Phys. Rev. E 90 (2014) 033203

[24] S. Wabnitz, B. Wetzel, Phys. Lett. A 378 (2014) 2750

[25] R. A. van Gorder, J. Phys. Soc. Jpn. 83 (2014) 054005

[26] A. Calini, C. M. Schober, Nat. Hazards Earth Syst. Sci. 14 (2014) 1431

[27] M. Onorato, A. R. Osborne, M. Serio, S. Bertone, Phys. Rev. Lett. 86 (2001) 5831 
[28] M. Onorato, A. R. Osborne, M. Serio, Phys. Rev. Lett. 96 (2006) 014503

[29] A. Chabchoub, N. Hoffmann, M. Onorato, N. Akhmediev, Phys. Rev. X 2 (2012) 011015

[30] V. E. Zakharov, A. A. Gelash, Phys. Rev. Lett. 111 (2013) 054101.

[31] A. A. Gelash, V. E. Zakharov, Nonlinearity 27 (2014) R1

[32] S. W. Xu, J. S. He, L. H. Wang, J. Phys. A 44 (2011) 305203

[33] G. Mu, Z. Y. Qin, J. Phys. Soc. Jpn. 81 (2012) 084001

[34] Z. Y. Qin and G. Mu, Phys. Rev. E 86 (2012) 036601 